\title{
High Frequency Oscillator Design Using a Single 45 nm CMOS Current Controlled Current Conveyor (CCCII+) with Minimum Passive Components
}

\author{
Mohd Yusuf Yasin, Bal Gopal \\ Department of Electronics and Communication Engineering, Integral University, Lucknow, India \\ E-mail:mmyasin@rediffmail.com \\ Received November 2, 2010; revised November 15, 2010; accepted February 18, 2011
}

\begin{abstract}
In the field of analog VLSI design, current conveyors have reasonably established their identity as an important circuit design element. In the literature published during the past few years, numerous application have been reported which are based on a variety of current conveyors. In this paper, an oscillator circuit has been proposed. This oscillator is designed using a single positive type second generation current controlled current conveyor (CCCII+). A CCCII has parasitic input resistance on it's current input node. This resistance could be exploited to reduce circuit complexities. Thus in this accord, a novel oscillator circuit is proposed which utilizes the parasitic resistance of the CCCII+ along with a few more passive components.
\end{abstract}

Keywords: Current Mode (CM) Circuit Applications, Current Conveyor (CC), Current Controlled Current Conveyor (CCC) Applications, CCCII Oscillator Circuit; Single CCCII+ Oscillator Circuit, Low Power Oscillator Circuit

\section{Introduction}

In the recent past, the analog VLSI has emerged as a promising technology for the future demands of low power and high bandwidth requirements. Current mode (CM) design approach is fast gaining in and establishing a trend setting reputation in the design of the modern day VLSI. It proves to be a viable technique that can help applying various design considerations which are ineffective or hard to apply otherwise. Because of it's superiority over the voltage mode approach, [1], the CM design approach appears to be a fit candidate for the next generation of analog VLSI.

Current mode design approach is one where circuits are operated on current stimuli and also the states of the circuits are represented in terms of currents rather than in terms of voltages. Current mode approach has numerous remarkable features, like, superior bandwidth, higher speed and better operational accuracy. It does not require highly sophisticated designs as demanded by the good performance VM amplifiers. Further, this approach can also manage with comparatively low precision design components. The CM design approach has been successfully applied to a variety of circuit applications. For example, several important CM applications are proposed in [2]. These applications are based on various CM devices like CCII, CCIII, CFA, OFC.

Current conveyor (CC) is an important and versatile current mode active building block, which has been considered superior to operational amplifiers [3], and can offer higher design flexibility than conventional operational amplifiers. It can be elegantly applied to a variety of analog circuit design problems. CC versions of various standard applications are found simpler and more elaborate than their voltage mode counterparts. In a CC, the voltage follower action between the input nodes and the current conveyance between input output nodes are the principal actions [1], and are controllable over a wide range [3]. A CC facilitates the use of both positive and negative feedbacks with equal ease, and can be used to simulate negative resistance. Current controlled current conveyor (CCC) is yet another CM active building block, where considerable parasitic resistance appears at the current input node and is controllable by the controlling (biasing) current. Both of these devices also offer the features of current duplication and current negation without any deterioration in the operation of the principal 
circuit. A brief review of the well known characteristics of an ideal CCCII+ are given in Equation (1) and Figure $\mathbf{1}[4,5]$.

$$
\left[\begin{array}{c}
I_{Y} \\
V_{X} \\
I_{Z}
\end{array}\right]=\left[\begin{array}{ccc}
0 & 0 & 0 \\
1 & R_{X} & 0 \\
0 & 1 & 0
\end{array}\right]\left[\begin{array}{c}
V_{Y} \\
I_{X} \\
V_{Z}
\end{array}\right]
$$

In a type CCCII+, both $I_{X}$ and $I_{Z}$ may flow into or out of the device simultaneously. For the other two possible combinations of these currents, the device is typed as CCCII-. The $R_{X}$ is input resistance at the node $X$ and depends upon the circuit structure.

In this work, an oscillator is realized using CMOS version of the CCCII+ shown in Figure 1. Resistance $R_{X}$ of the CCCII+ is exploited and is treated like any other passive resistor with the aim of reducing the demand of external passive resistors.

\section{Oscillator Circuit Scheme}

Several oscillator circuits have been published recently $[4,6-10]$. These circuits have invariably been proposed on the basis of the multiple use of CC/CCC along with two or more passive components, incorporating both inverting and non inverting outputs, and, in some cases, a few more design building blocks, etc. A number of oscillator topologies are presented in [11]. ICCII is the active device used in all these topologies. However, the minimum passive component count is irredundantly four, along with at least one ICCII.

Here in this work, a novel scheme is proposed as depicted in Figure 2. This scheme is quite simple and employs only one single output $\mathrm{CCCII}+$ with a possible minimum passive component count. Here the basic circuit structure is proposed with four passive components, however, the subsequent analysis helps in reducing the external component count to three. The circuit also has the added features like, very low power requirement,

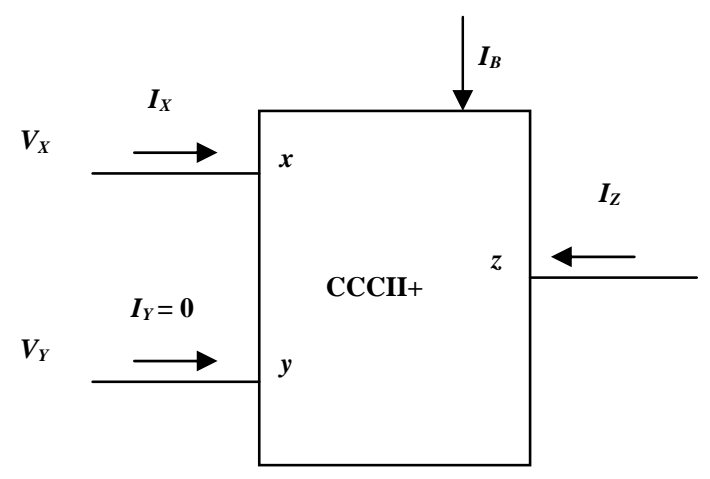

Figure 1. Block Diagram representation of the $2^{\text {nd }}$ generation CCCII+.

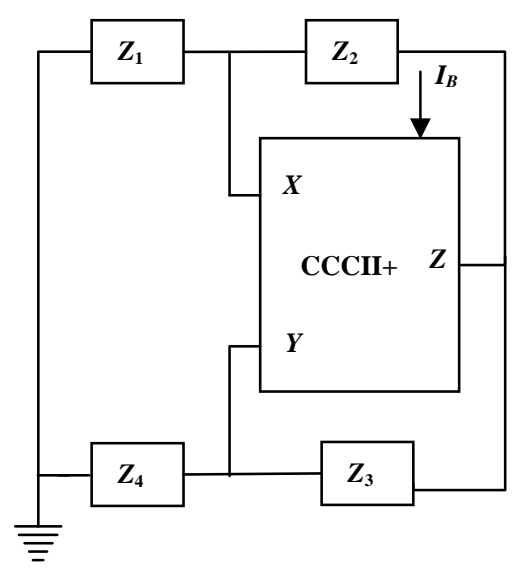

Figure 2. Generalized scheme for the proposed Oscillator. All $Z_{i}$ 's are impedances.

capability of generating high oscillation frequency, low turn-on time [6], and electronically tunable frequency through $I_{B}$.

Routine analysis of the scheme of Figure 2, gives the following characteristic equation:

$$
\begin{aligned}
& Z_{2} Z_{4}=Z_{4} R_{X}+Z_{1} Z_{2}+2 Z_{1} Z_{3} \\
& +R_{X}\left(Z_{1}+Z_{2}+Z_{3}\right)
\end{aligned}
$$

For a real frequency of oscillation, and the gain adjustment, a suitable second order polynomial is required, so that the real and imaginary components yield. This requirement of Equation (2) can be fulfilled for the following specific choice of external components as given in Equation (3). Arrows in Equation (3) indicate the operation of replacing the impedances by the corresponding passive components (for example, impedance $Z_{1}$ is replaced by the capacitor $C_{1}$ ).

$$
Z_{1} \rightarrow C_{1} ; Z_{2} \rightarrow C_{2} ; Z_{3} \rightarrow R_{3} ; Z_{4} \rightarrow R_{4}
$$

The choice of Equation (3) is incorporated in the scheme of Figure 2 and the following s-domain characteristic polynomial is obtained.

$$
\begin{aligned}
& s^{2} C_{1} C_{2} R_{X}\left(R_{3}+R_{4}\right)+ \\
& s\left(C_{1} R_{X}+C_{2} R_{X}+2 C_{2} R_{3}-C_{1} R_{4}\right)+1=0
\end{aligned}
$$

Equation (4) gives the necessary gain condition and the frequency of oscillations.

$$
\begin{gathered}
C_{1} R_{X}+C_{2} R_{X}+2 C_{2} R_{3}=C_{1} R_{4} \\
\omega_{O}=\frac{1}{\sqrt{C_{1} C_{2} R_{X}\left(R_{4}+R_{3}\right)}}
\end{gathered}
$$

Equations (5) and (6) clearly show the insignificance of $R_{3}$. If it is shorted, $\left(R_{3}=0\right)$, both the Equations (5) and (6) are further simplified.

$$
R_{4}=2 R_{X}
$$




$$
\omega_{O}=\frac{1}{\sqrt{2} C R_{X}}
$$

Thus it is possible to realize the oscillator using a single $\mathrm{CCCII}+$, one Resistor and two equal capacitors. The final complete circuit is presented in Figure 3.

On the basis of Figure 3 and the Equations (7) and (8), a few observations are worth noting. Equation (7) depicts ideal condition, and thus Equation (8) remains a valid equation when expressed in terms of $R_{4}$. Further, from Figure 3, the feedback signal with respect to node $Y$ is $V_{2}=\frac{R_{X}-R_{4}-s C R_{X} R_{4}}{R_{4}\left(1+2 s C R_{X}\right)} V_{3}$. For large values of $R_{4}$ and $\omega, \frac{V_{2}}{V_{3}} \rightarrow-1 / 2$. It clearly indicates that a larger value of $R_{4}$ is necessary to build up the required level of the $Y$ node feed back signal and $180^{\circ}$ phase shift so that the circuit sustains oscillations. Therefore the oscillation frequency should better be defined using Equation (8) instead of using the suggestion of Equation (7), as it predicts only the ideal condition for oscillations. Simulation results also suggest the independence of the frequency of oscillations of $R_{4}$.

Ignoring the body effect, the estimate of resistance $R_{X}$ of the above circuit, is given by $R_{X} \approx \frac{1}{g_{m 10}+g_{m 9}}$. For matched transistors $\mathrm{M} 9$ and M10, $R_{X} \approx \frac{1}{\sqrt{8 \beta I_{B}}}, \beta$ being the device transconductance of M9 [5].

\section{Circuit of the CCCII+}

For realization of the above oscillator, the class $\mathrm{AB}$ CCCII+ circuit adopted is shown in Figure 4, and is readily available in literature. It's bipolar version is

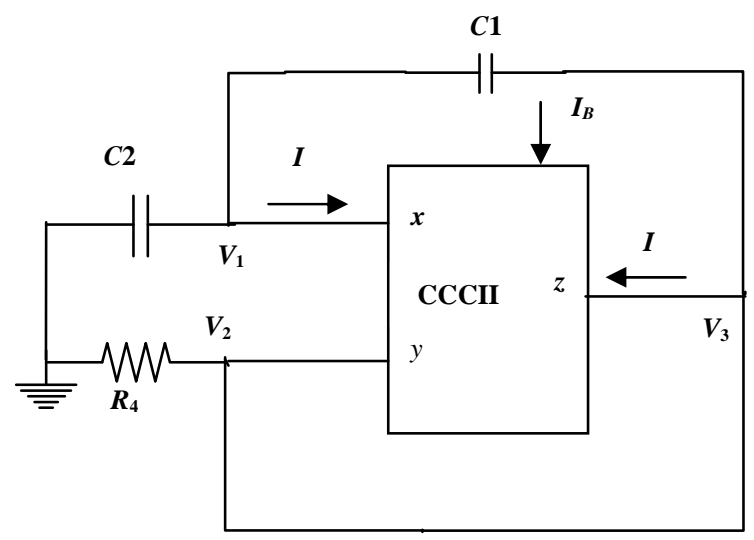

Figure 3. Simplified circuit schematic of the CCCII+ based oscillator.

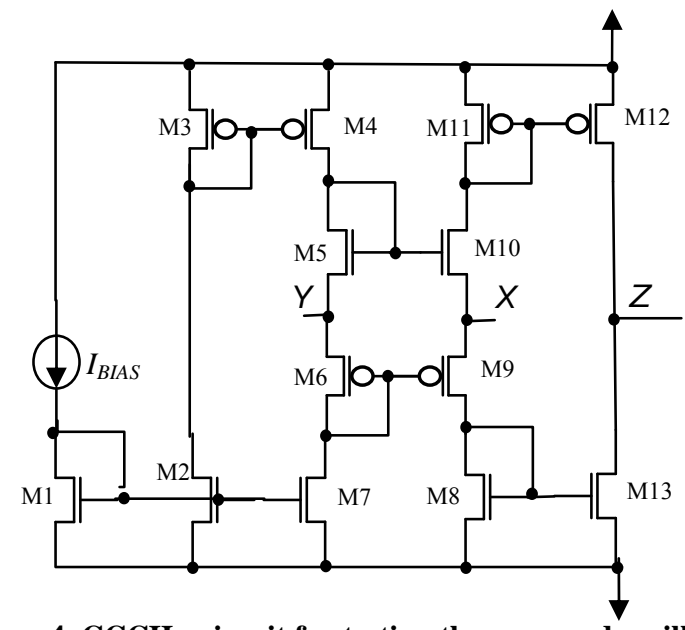

Figure 4. CCCII+ circuit for testing the proposed oscillator. Upward arrow is to $V_{D D}$ and the downward arrow is to $V_{S S}$.

studied by many authors, $[12,13]$. It's CMOS version can be found in references $[5,10]$. In the present work, this circuit is redesigned in $45 \mathrm{~nm}$ CMOS and is simulated using the "Predictive Technology Model Beta Version 45 nm MOS Parameters" compatible with HSPICE [14]. The design details of this circuit are presented in Table 1.

\section{Verification and Results}

CCCII+ of Figure 4 is designed in $45 \mathrm{~nm} \mathrm{CMOS,} \mathrm{and} \mathrm{is}$ applied to the realization of the proposed oscillator.

The application is then simulated on HSPICE and the performance of the oscillator (node $Z$ voltage signal) is presented in Figure 5. For clarity of the necessary details, Figure 5 is windowed between $600 \mathrm{~ns}$ and $800 \mathrm{~ns}$; and is presented in Figure 6. However, the simulation is done for the entire $0.1 \mathrm{~ms}$ interval.

Fourier analysis, with respect to the principal frequency $(38.48 \mathrm{MHz})$, is performed on the node $Z$ signal to ascertain the quality of the oscillations. Result of this analysis is presented in Figure 7. Peaks in Figure 7 correspond to the principal frequency of the oscillator and it's harmonic frequencies. Third harmonic component is significant $(-27 \mathrm{~dB})$ compared to the second harmonic component $(-30 \mathrm{~dB})$. Estimates of the total harmonic distortion (THD) and the DC component of the node $Z$ signal are important quality matrices. Both these parameters are found reasonably very low. The simulation results are summarized in Table 2 .

It is noteworthy that the sustainable oscillations are established without requiring a trigger signal. Further, the value of resistor $R_{4}$ estimated by Equation (6) above, would theoretically establish oscillations. It is observed that higher values of $R_{4}$ enhance the voltage buildup 
Table 1. Design details of the circuits of Figure 3 \& Figure 4.

\begin{tabular}{ll}
\hline Design Parameter & Value \\
\hline$R_{4}$ & $15 \mathrm{k} \Omega$ \\
$C_{1}=C_{2}$ & $1 \mathrm{pF}$ \\
Supply Voltage & $\pm 1.0 \mathrm{~V}$ \\
$\mathrm{I}_{\mathrm{BIAS}}$ & $3 \mu \mathrm{A}$ \\
$W / L$ & $0.98 \mu \mathrm{m} / 0.2 \mu \mathrm{m}$ (all NMOS) \\
$W / L$ & $8.3 \mu \mathrm{m} / 0.36 \mu \mathrm{m}($ all PMOS $)$ \\
Parameters & $45 \mathrm{~nm}(\beta$ version $)$, HSPICE \\
\hline
\end{tabular}

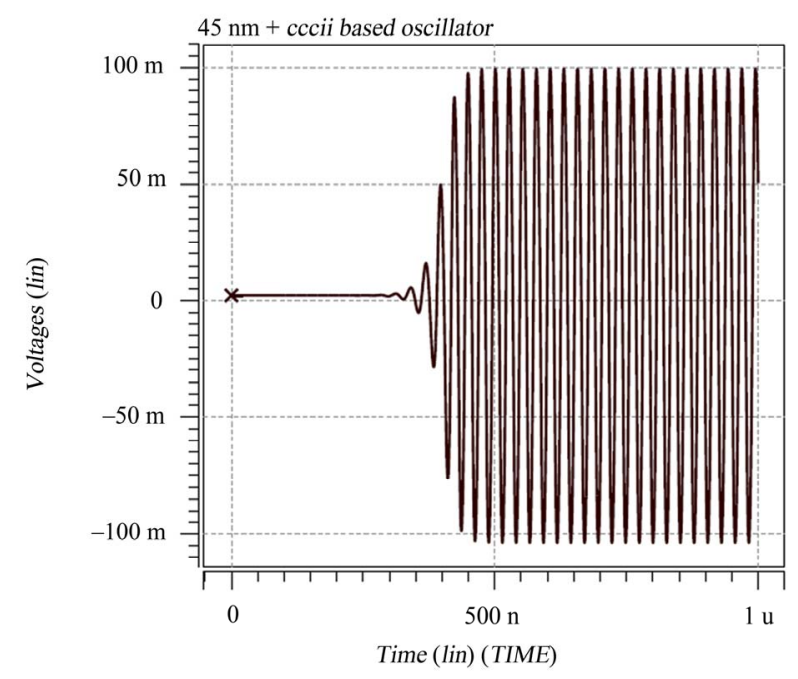

Figure 5. Output of the proposed oscillator (node $Z$ ). $I_{B}=$ $3 \mu \mathrm{A}, C=10 \mathrm{pF}$. Figure shows startup time $\approx 400 \mathrm{~ns}$.

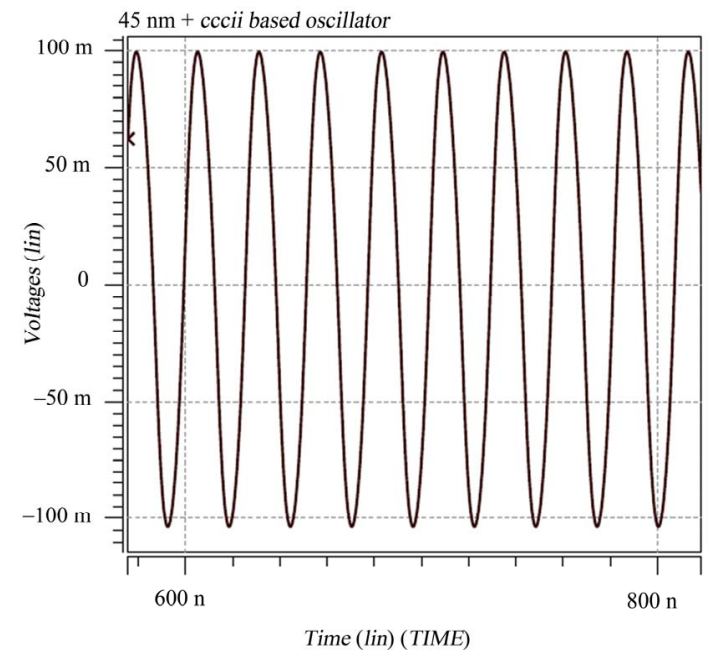

Figure 6. Expanded view of the output signal (node $Z$ ) in Figure 5.

quicker and hence the oscillations start up earlier. It is also observed that $R_{4}$ do not affect the oscillation fre-

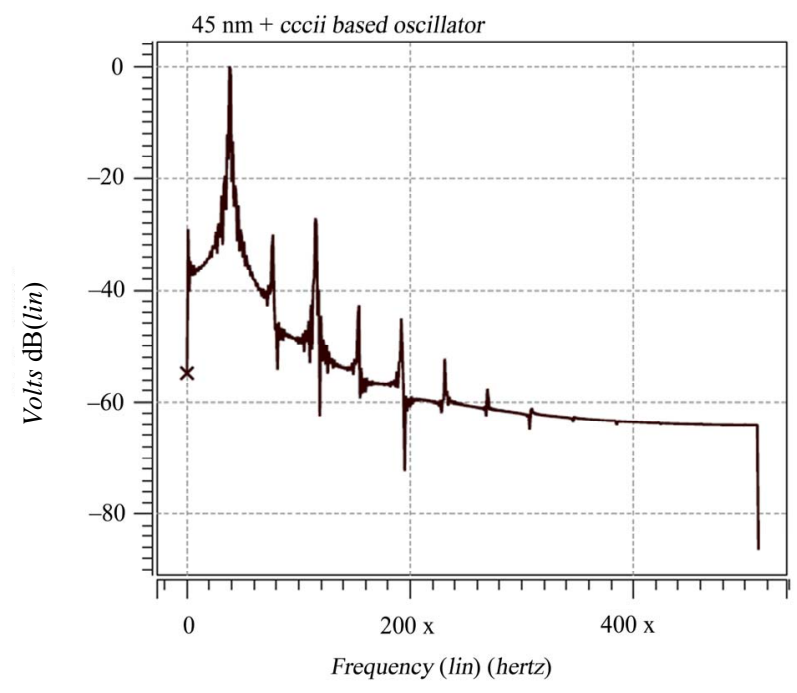

Figure 7. Fourier analysis of the signal at node $\mathrm{z}$ of Figure 5. First peak appears at $38.5 \mathrm{MHz}$. Subsequent peaks occur at harmonic frequencies.

Table 2. Performance results of the proposed oscillator.

\begin{tabular}{ll}
\hline Performance Parameters & Detail \\
\hline Frequency & $38.5 \mathrm{MHz}$ \\
THD & $-31.4 \mathrm{~dB},(2.7 \%)$ \\
DC Component & $-1.8 \mathrm{mV}$ \\
Peak Average Magnitude & $-104 \mathrm{mV}$ to $99.4 \mathrm{mV}$ \\
Total Power Dissipation(biasing & $257 \mu \mathrm{W}$ \\
source) & $\sim 400 \mathrm{~ns}$ \\
Oscillations start-up Time & $-0.75 \mathrm{~dB}$ \\
SNR at output node &
\end{tabular}

quency though Equation (8) can be expressed in terms of $R_{4}$. This is merely because of Equation (7).

Authors of reference [4] reported frequency of operation in $\mathrm{kHz}$ range for their proposed oscillators with a THD of $0.5 \%$ using $\mathrm{CC} / \mathrm{CCC}$ based upon bipolar technology.

Authors of reference [11] use $1.2 \mu \mathrm{m}$ CMOS based ICCII using $\pm 2.5 \mathrm{~V}$ supply voltages. The total active area for the proposed ICCII was $2096 \mu \mathrm{m}^{2}$. Also the proposed oscillators gradually build up to final peak to peak amplitude of oscillations in about $400 \mu \mathrm{s}$. Test results in [11] are presented for $39.78 \mathrm{kHz}$.

The circuit scheme proposed here in this work is a low voltage, low power scheme, based on CCCII designed in $45 \mathrm{~nm}$ CMOS technology, biased at $\pm 1 \mathrm{~V}$ and simulation results are summarized in Table 2 . In addition, the proposed circuit can generate frequencies up to $100 \mathrm{MHz}$ (at $I_{B}=2.79 \mu \mathrm{A}, C_{1}=C_{2}=0.225 \mathrm{pf}$ ), and requires only $19.6 \mu \mathrm{m}^{2}$ active area, which is quite small [11]. 
Figure 8 shows a logarithmic plot for frequency variation with respect to capacitance. The graph shows a natural trend of as frequency drops with increasing capacitance.

In Figures 9 a plot for frequency variation with biasing current of the CCCII+ is presented on logarithmic scale. Simulation results show that a variation in the bias current, $\Delta I_{B}=2.9 \mu \mathrm{A}(0.4 \mu \mathrm{A}$ to $3.3 \mu \mathrm{A})$, cause the oscillator frequency to vary as $\Delta f=25.78 \mathrm{MHz}(15.2 \mathrm{MHz}$ to $40.98 \mathrm{MHz}$ ). For the sake of analysis, a figure of merit could be defined as the current to frequency transfer coefficient, $K_{f i}$, [15]. Thus for $C_{1}=C_{2}=1 \mathrm{pf}, K_{f i N}=25.78 \mathrm{MHz}$ $/ 2.9 \mu \mathrm{A}=8.9 \mathrm{MHz} / \mu \mathrm{A} . K_{f-I}$ depends on capacitances and the biasing current. It varies directly with the $I_{B}$ and inversely with the capacitances.

\section{Non Idealities of CCCII+ and Their Impact on Circuit Performance}

In the above analysis, the CCCII+ is considered ideal. However, a number of non-idealities are present in a practical CCCII+. Considering some of these nonidealities, the device model of the CCCII+ of Figure 1 can be described as below:

$$
\begin{gathered}
I_{Z}= \pm \alpha I_{X} \\
V_{X}=I_{X} R_{X}+\beta V_{Y} \\
I_{Y}=I_{\delta}
\end{gathered}
$$

where $\alpha$ in Equation (9), is the current conveyance coefficient between nodes $X$ and $Z ; \beta$ in Equation (10), is the voltage gain from node $Y$ to node $X$ and is usuall $y<1$. $I_{\delta}$ in Equation (11) is the input current at node $Y$. For analytical simplicity in the proposed oscillator scheme, it is assumed that this current is a function of the voltage at node $Z$. Therefore, $I_{\delta}=V_{Z} / R_{\delta}$, where $R_{\delta}$ is the corre-

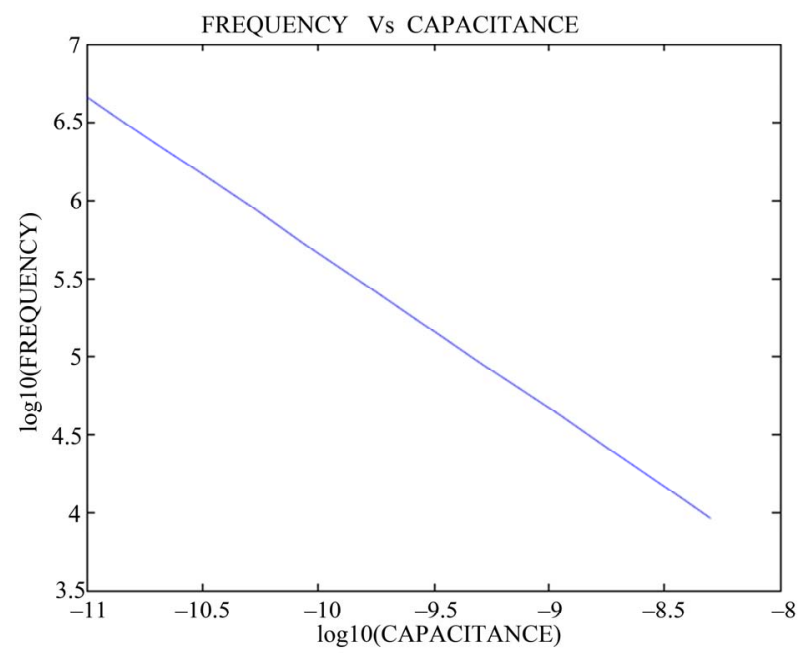

Figure 8. Frequency variation with Capacitor $\left(C_{1}=C_{2}=C\right)$.

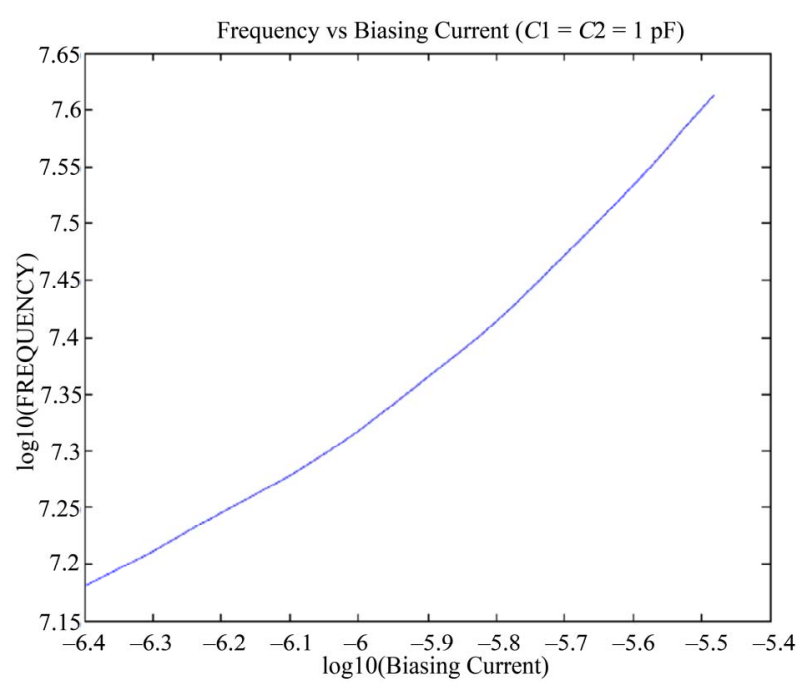

Figure 9. Frequency variation with biasing current. Case $2: C=1 \mathrm{pF}$.

sponding resistance at the $Y$ node. Also, as usual, $C_{1}=C_{2}$ $=C$. Using these assumptions, analysis of circuit in Figure 3 , gives the following modified characteristic equation:

$$
\begin{aligned}
& s^{2} C^{2} R_{4} R_{X}+ \\
& s C\left(2 R_{X}-2 \alpha \beta R_{4}+R_{4}+\beta R_{4}-\alpha R_{4} R_{X}+2 \frac{R_{4}}{R_{X}}\right) \\
& +\left(1+\frac{R_{4}}{R_{\delta}}\right)=0
\end{aligned}
$$

On solving Equation (12), the results are:

$$
\begin{gathered}
\frac{1}{R_{4}}+\frac{1}{R_{\delta}}=\frac{\alpha(1+2 \beta)-(1+\beta)}{2 R_{X}}=\frac{K}{2 R_{X}} \\
\omega^{2}=\frac{\frac{1}{R_{\delta}}+\frac{1}{R_{4}}}{C^{2} R_{X}}=\frac{K}{2 C^{2} R_{X}^{2}} \\
\omega=\sqrt{K} \frac{1}{\sqrt{2} C R_{X}}=\sqrt{K} \omega_{O} \\
\frac{\omega}{\omega_{O}}=\sqrt{K}<1
\end{gathered}
$$

Equation (13) relates $R_{4}, R_{X}$, and $R_{\delta}$. Equation (15) shows a possible elimination of $R_{\delta}$, Hence either of $R_{4}$ and $R_{\mathrm{X}}$ or both may get modified on account of the voltage and current tracking errors of the CCCII. Here it is assumed that the circuit non-idealities do not cause significant change in the value of $R_{X}$. Equation (16) shows that the oscillation frequency $\omega$ under non-ideal conditions is lesser than the ideal oscillation frequency $\omega_{0}$. Further more,

$$
K=\alpha(1+2 \beta)-(1+\beta)
$$


Equation (17) indicates that the variation in the oscillator frequency depends on the current and voltage tracking errors, and shows no effect of the current existing at node $Y$ of the CCCII+. Percent decrease in the frequency can be described as:

$$
\frac{\omega_{O}-\omega}{\omega_{O}}=1-\sqrt{K}
$$

For an ideal situation, $\alpha \rightarrow 1, \beta \rightarrow 1, I_{\delta}=0$ and $R_{\delta} \rightarrow \infty$; hence Equation (13) reduces to $\frac{1}{R_{4}}=\frac{1}{2 R_{X}}$, and Equation (14) reduces to Equation (8), and hence from Equations (16) and (18), $\omega=\omega_{0}$. But for a $5 \%$ tracking error in the values of $\alpha$, and $\beta$, e.g. $\alpha=0.95, \beta=0.95$, using Equation (18), the deviation in the oscillation frequency is observed to be $\frac{\omega_{O}-\omega}{\omega_{O}}=0.1028$, or $10.3 \%$.

\section{Time Domain and Stabilty Considerations}

The time domain analysis may be significant in giving better insight in the functioning and performance of the oscillator circuit. Assuming the circuit of Figure 3 relaxed, it can be described by the following system of equations for $v_{1}(t)$ and $v_{3}(t)$ voltages of nodes 1 and 3 respectively

$$
\begin{aligned}
& \left(C R_{X}\right)^{2} R_{4} \frac{d^{2} v_{1}}{d t^{2}}+C R_{X}\left(2 R_{X}-R_{4}\right) \frac{d v_{1}}{d t}+R_{X} v_{1}=0 \\
& \left(C R_{X}\right)^{2} R_{4} \frac{d^{2} v_{3}(t)}{d t^{2}}+C R_{X}\left(2 R_{X}-R_{4}\right) \frac{d v_{3}(t)}{d t} \\
& -R_{X} v_{3}(t)=0
\end{aligned}
$$

Equation (19) predicts oscillatory behaviour for the option $R_{4}=2 R_{x}$ as has already been indicated above.

Using this option, and defining $\omega_{o}^{\prime}=\frac{1}{C R_{4}}$, general solutions of Equations (19) and (20) are as follows

$$
\begin{aligned}
& v_{1}(t)=c_{1} e^{j \omega_{0}^{\prime} t}+c_{2} e^{-j \omega_{0}^{\prime} t} \\
& v_{3}(t)=c_{3} e^{\sqrt{2} \omega_{0}^{\prime} t}+c_{4} e^{-\sqrt{2} \omega_{0}^{\prime} t}
\end{aligned}
$$

In the above Equations (21) and (22), the coefficients $C_{1}, C_{2}, C_{3}$, and $C_{4}$, are arbitrary constants. Equation (21) is oscillatory in nature. In Equation (22), one of the terms rises exponentially to saturation while the other term sharply decays out for large $\omega^{\prime}$ and hence oscillations attain their amplitude. $\omega_{o}=\sqrt{2} \omega_{o}^{\prime}$

Again one can consider the gain limits of the circuit. For this purpose, feedback signal from node $Z$ to node $X$ is through $C_{1}$ and $C_{2}\left(C_{1}=C_{2}\right)$, while to node $Y$ is through $R_{4}$. Thus the gain function corresponding to the capacitive feedback arm is:

$$
\begin{gathered}
\frac{v_{1}(s)}{v_{3}(s)}=\frac{s C R_{X}+1}{s 2 C R_{X}+1}=\frac{1}{2} \frac{s+\frac{2}{C R_{4}}}{s+\frac{1}{C R_{4}}} \\
=\frac{1}{2} \frac{s+2 \omega_{o}^{\prime}}{s+\omega_{o}^{\prime}} \\
\left|\frac{v_{1}(s)}{v_{3}(s)}\right|=\frac{1}{2} \frac{\sqrt{\omega^{2}+4 \omega_{o}^{\prime 2}}}{\sqrt{\omega^{2}+\omega_{o}^{\prime 2}}}=\frac{1}{2} \frac{\sqrt{\omega_{n}^{2}+4}}{\sqrt{\omega_{n}^{2}+1}}
\end{gathered}
$$

Similarly the gain function corresponding to the resistive arm is

$$
\begin{gathered}
\frac{v_{3}(s)}{v_{1}(s)}=\frac{\left(s C R_{X}-1\right) R}{s C R R_{X}+R_{X}-R}=\frac{s-\frac{2}{C R_{4}}}{s-\frac{1}{C R_{4}}}=\frac{s-2 \omega_{o}^{\prime}}{s-\omega_{o}^{\prime}} \\
\left|\frac{v_{3}(s)}{v_{1}(s)}\right|=\frac{\sqrt{\omega^{2}+4 \omega_{o}^{\prime 2}}}{\sqrt{\omega^{2}+\omega_{o}^{\prime 2}}}=\frac{\sqrt{\omega_{n}^{2}+4}}{\sqrt{\omega_{n}^{2}+1}}
\end{gathered}
$$

Equations (24) and (26) are described as a function of normalized frequency, $\omega_{n}=\omega / \omega_{o}^{\prime}$. From Equation (24), the gain limits are: for $\omega_{n} \rightarrow 0,\left|\frac{v_{1}(s)}{v_{3}(s)}\right| \rightarrow 1$ and $\omega_{n} \rightarrow \infty,\left|\frac{v_{1}(s)}{v_{3}(s)}\right| \rightarrow \frac{1}{2}$. Similarly from Equation (26), $\omega_{n} \rightarrow 0,\left|\frac{v_{3}(s)}{v_{1}(s)}\right| \rightarrow 2$ and $\omega_{n} \rightarrow \infty,\left|\frac{v_{3}(s)}{v_{1}(s)}\right| \rightarrow 1$. In both cases, the gain transitions occur between $1<\omega_{n}<2$. However, the phases for Equations (23) and (25), being opposite to one another, start at $0^{\circ}$ phase angle, both attain a peak $\left(\omega_{n} \approx 1.4, \varphi(\omega) \approx \pm 19.47^{\circ}\right.$ and then gradually die towards zero individually. It is thus concluded that the system is quite stable [16].

\section{Conclusions}

In this work, a novel oscillator is designed using a single $\mathrm{CCCII+}$, two passive capacitors to control frequency and one passive resistor to sustain the necessary gain. The simulation results of the oscillator verify the circuit capability to generate megahertz oscillations. Quality of oscillations is also reasonable as per the simulation results presented in Figures $\mathbf{5}$ and $\mathbf{6}$, summarized in Table 2. The DC component of the output is observed about $-1.8 \mathrm{mV}$ and the total harmonic distortion in the output 
node $Z$ signal is about $2.7 \%(-31.4 \mathrm{~dB})$ at $38.5 \mathrm{MHz}$ frequency (see Table 2). The peak to peak amplitude of the output voltage is $203 \mathrm{mV}$. Also, the simulation shows the average power dissipation low, $257 \mu \mathrm{W}$ when biased through $\pm 1.0 \mathrm{~V}$ and a $3 \mu \mathrm{A}$ source. The oscillator is also investigated for higher frequencies and found capable of generating $100 \mathrm{MHz}$ at $I_{B}=2.79 \mu \mathrm{A}, C_{1}=C_{2}=0.225 \mathrm{pf}$ satisfactorily. It is also supported by the Figures 8 and 9 that smaller capacitance and larger bias current results higher frequency oscillations.

Further more, it is noticeable that a higher value of $R_{4}$ is required to set in the oscillations. The reasons may include

1) Requirement of the feedback loop gain to satisfy the criterion of oscillations.

2) Current, and voltage follow up errors at the relative node pairs $(Z, X)$ and $(Y, X)$ respectively. If $R_{X}$ assumed unchanged, critical value $\mathrm{R}_{4}$ requires an upward modification on account of $\alpha \neq 1, \beta \neq 1$.

In presence of such non-idealities, however, the model of CCCII described in Equation (1) may be modified to accommodate the tracking errors and the voltage node input current.

$$
\left[\begin{array}{c}
I_{Y} \\
V_{X} \\
I_{Z}
\end{array}\right]=\left[\begin{array}{ccc}
0 & 0 & 0 \\
\beta & R_{X} & 0 \\
0 & \pm \alpha & 0
\end{array}\right]\left[\begin{array}{c}
V_{Y} \\
I_{X} \\
V_{Z}
\end{array}\right]+\left[\begin{array}{l}
1 \\
0 \\
0
\end{array}\right]\left[I_{\delta}\right]
$$

It is also noticeable that it is the deviations (the absolute values of the coefficients $\alpha$ and $\beta$ ) that affect the results much more than the input current or impedance of the node $Y$ as is clearly indicated by Equation (15). It is further noteworthy that the definition of the parasitic resistance in Equation (1) includes both gate transconductance and body transconductance of M9 and M10. Body transconductnace of the MOSFETs, was ignored in the analysis. Inclusion of the body transconductance of the MOSFETs, however, shows a favorable impact on decreasing $R_{X}$, and thus improves the oscillator performance.

\section{References}

[1] S. Sedra, et al., "The Current Conveyor: History, Progress and New Results," IEE Proceedings (Part G) of Circuits, Devices and Systems, Vol. 137, No. 2, April 1990, pp. 78-87. doi:10.1049/ip-g-2.1990.0015

[2] S. S. Rajput, et al., "Advanced Applications of Current
Conveyors: A Tutorial," Journal of Active and Passive Electronic Devices, Vol. 2, No. 2, 2007, pp. 143-164.

[3] J. Zhao, et al., "Design of Tunable Biquadratic Filters Employing CCCIIs: State Variable Block Diagram Approach," Analog Integrated Circuits and Signal Processing, Vol. 62, No. 3, March 2010, pp. 397-406.

doi:10.1007/s10470-009-9348-0

[4] N. Pandey, et al., "Sinusoidal Oscillator-A New Configuration Based on Current Conveyor," Proceedings of XXVII General Assembly of International Union of Radio Science (URSI), Delhi, 23-29 October 2005, pp 23-29.

[5] M. Siripruchyanun, "A Temperature Compensation Technique for CMOS Current Controlled Current Conveyor (CCCII)," Proceedings of ECTI-CON 2005, North Bangkok, 12-13 May 2005, pp. 510-513.

[6] S. B. Salem, et al., "A High Performances CMOS CCII and High Frequency Applications," Analog Integrated Circuits and Signal Processing, Vol. 49, No. 1, October 2006, pp. 71-78. doi:10.1007/s10470-006-8694-4

[7] J. Horng, et al., "Sinusoidal Oscillators Using Current Conveyors and Grounded Capacitors," Journal of Active and Passive Electronic Devices, Vol. 2, No. 2, 2007, pp. 127-136.

[8] W. Kiranon, et al., "Current Controlled Oscillator Based on Translinear Conveyors," Electronics Letters, Vol. 32, No. 15, 1996, pp. 1330-1331. doi:10.1049/el:19960936

[9] W. Kiranon, et al., "Electronically Tunable Multifunction Translinear-C Filter and Oscillator," Electronics Letters, Vol. 33, No. 7, 1997, p. 573. doi:10.1049/el:19970382

[10] J. W. Horng, "A Sinusoidal Oscillator Using Current-Controlled Current Conveyor," International Journal of Electronics, Vol. 88, No. 6, 2001, pp. 659-664. doi: $10.1080 / 00207210110044369$

[11] A. Toker, et al., "New Oscillator Topologies Using Inverting Second-Generation Current Conveyors," Turkish Journal of Electriial Engineering \& Computer Science, Vol. 10, No. 1, 2002, pp. 119-130.

[12] E. Yuce1, et al., "Universal Resistorless Current-Mode Filters Employing CCCIIs," International Journal of Circuit Theory and Applicaitons, Vol. 36, No. 5-6, 2008, pp. 739-755.

[13] T. Parveen1, et al., "A Canonical Voltage Mode Universal CCCII-C Filter," Journal of Active and Passive Electronic Devices, Vol. 4, No. 1-2, 2009, pp. 7-12.

[14] Predictive Technology Model, 2006. http://ptm.asu.edu

[15] S. Soclof, "Design and Applications of Analog Integrated Circuits," Prentice Hall of India, Delhi, 2004.

[16] G. Daryanani, "Principles of Active Network Synthesis and Design," John Wiley and Sons, New York, 1976. 